\title{
Investigative study on the bacteriological, physical and chemical profiles of aquaculture waters: insights into health hazards for fish and human
}

\author{
Abdulkadir A., ${ }^{1}$ Abubakar, M.I. ${ }^{2}$ and Abdulkadir O.J., ${ }^{3}$ \\ 1,2,3, Department of Aquaculture and Fisheries, Faculty of Agriculture, University of Ilorin, \\ Ilorin, Nigeria.
}

\begin{abstract}
Bacteriological and physic-chemical analysis of fish pond water is very important in aquaculture as this gives insights into likely threats to aquaculture and associated personnels. Bacteriological and physico-chemical profiles of selected fish ponds in the Ilorin West area of Kwara State, Nigeria were investigated to evaluate the water quality of rearing enclosures. Physico-chemical analyses revealed quality parameters were within the recommended range for aquaculture. Following bacteriological analyses of static water pond culture, the TVC and TCC showed temporal variations with concentration increasing with sampling time. However, the FCC showed fluctuation. Totally, 8 bacteria groups were isolated from both rearing enclosures. Of these, Gram negative bacteria showed dominance. In which 5 Gram negative (Escherichia coli, Proteus spp, Serratia spp, Enterobacter spp and Pseudomonas spp) and 3 Gram positive (Staphylococcus spp, Streptococcus spp, and Bacillus spp) were encountered. Estimates of bacteria occurrence in both rearing facilities respectively gave : Staphylococcus spp (20\%), Streptococcus spp (12\%) Proteus spp (8\%) Enterobacter spp (20\%) Serratia spp (16\%), Bacillus spp (9\%), Escherichia coli (8\%), Pseudomonas spp (7\%) from earthen pond water sampled. While Staphylococcus spp (18\%), Streptococcus spp (16\%), Proteus spp (8\%), Enterobacter spp (22)\%, Serratia spp (8\%), Bacillus spp (15\%), Escherichia coli (8\%), Pseudomonas spp (6\%) from concrete water sampled. Conclusively, although there is the presence of bacteria groups of public health concern, the static water exchange provides benefits of natural processing of wastes and restoration of the pond ecosystem. Notably, the presence of Escherichia coli gives indication of presence of pathogenic organisms of enteric origin. The presence of these organisms has been associated with a lack of tentative pond management and effective biosecurity procedures. One recommendation to this culture system (static water aquaculture) is the consideration of adaptation of concepts in biomimicry or biofloc technology which operates on similar principles.
\end{abstract}

Keywords: pathogens, water quality, biofloc, stagnant aquaculture, bacteria, public health 


\section{Introduction}

One single most significant factor devastating the aquaculture industries is disease. For example, in the shellfish industry, an estimate revealed US\$8-15 billion shellfish value was lost globally to a single disease agent, white spot syndrome virus (WSSV) (Lightner et al., 2012). Likewise global losses associated with other OIE listed viruses were documented; infectious hypodermal and haematopoietic virus, IHHV (US\$1billion); Yellow head virus (YHV), US\$0.5billion; Taura syndrome virus, (TSV), US\$3billion (Stentiford et al., 2012). Yet, unlike other livestock production, the aquatic environment presents a limitation to biosecurity. That is, due to the open nature of this environment- sometimes with exception of closed systems, such as recirculatory aquaculture - total exclusion of pathogens may be impractical (Georgiades et al., 2016; Hasimuna et al., 2020). And in the presence of stressor(s), the health of the stock may be under jeopardy (Eissa \& Wang, 2016) which can devastate the economical, ethical and environmental sustainability of aquaculture. For instance, in Ecuador, loss due to WSSV outbreaks was around $63,000 \mathrm{mt}$ valued at US\$280.5million, and it was estimated that up to 23,000people had been laid off (Alday de Graindorge \& Griffith, 2001). In addition to this concern, aquatic environments have been implicated to represent reservoirs of pathogenic organisms which may pose risks to human health (Islam et al., 1993; Dekić et al., 2018). A good indication of presence of pathogenic bacteria are common attributes to faecal coliform bacteria, for example, the Escherichia coli and enterococci (Tyagi et al., 2006).

Moreover, since pathogenic colonisation of aquatic organisms can depend on concentration of pathogens (Dekić et al., 2018), of particular concern is the aquaculture practicing zero-water exchange. In which present pathogenic challenge may increase to the level capable of overwhelming the stock defense system (Arias-Moscoso et al., 2018) thereby leading to the establishment of pathogens. Subsequently, this water is discharged to the surrounding environment which further poses risk to the wild population or threats to human use (Vouga \& Greub, 2016). In Nigeria, for example, a majority of the aquaculture production is pond-based (Akankali et al., 2011) and due to limited water supply, these aquaculture usually involve nearly/zero water exchange. For example, according to a study in Nigeria, 35.7\% surveyed farms practiced stagnant water aquaculture, with $48.2 \%$ practicing throughflow systems and only $16.1 \%$ adopting water recirculatory systems (Emmanuel et al., 2014). Several lines of evidence have demonstrated increased pathogens may pose risks to the surrounding environments (Alaliyat et al., 2019; Shea et al., 2020). For example, the probability of detecting pathogen environmental DNA was 2.72 times greater at active farms compared to inactive farms (Shea et al., 2020). To the best of the authors' knowledge, the majority of studies have based their exploration on the bacteria profiles and the antibiotic susceptibility tests (e.g., Nzeh \& Udeze, 2012; Njoku et al., 2015; Fakorede et al., 2019) however disregarding the comparisons of sampling times and changes to bacteria profiles. Therefore, in order to give insight into the status of health of aquaculture settings practicing stagnant water aquaculture, this study set out to investigate the microbial profile, particularly bacteria, and quality of pond waters used for aquaculture in the study area. With consideration of temporal variations in bacteria concentration. The outcomes of which may serve to extrapolate conditions of other regions - especially those adopting similar management practices.

\section{Materials and Methods}

The study area is within Kwara state, Nigeria, and is characterised with varieties of economic activities. Aquaculture operations is one major agricultural activity conducted in this area, with most adopting pond water aquaculture with some incorporating concrete culture during production stages. The most cultured species is the African catfish (Clarias gariepinus). Notably, the majority of aquaculture operations are aggregated into farm 'estates' with different people operating on the average 2 ponds/ individuals. Meaning different 'estates' adopt similar management practices and are exposed to limited variation in environmental conditions. 


\section{Sample collection}

Water samples were collected from five fish farms (two water holding facilities, an earthen pond and a concrete tank from each farm) using sterile bottles from below water level approximately $10-15 \mathrm{~cm}$ below the surface (Njoku et al., 2015). The water was transported to the Microbiology laboratory in the department of Microbiology, University of Ilorin in an ice-packed container for bacteriological analysis. While the other important information about the ponds such as source of water supply, period or length of use was obtained by interview.

\section{Determination of water quality parameters}

The physical and chemical parameters of the pond water were measured within the holding time of each parameter following standard methods.

\section{Determination of pH, temperature, electrical conductivity, DO and TDS}

Water temperature $\left({ }^{\circ} \mathrm{C}\right)$, Total dissolved solids (TDS, $\left.\mathrm{mg} / \mathrm{l}\right), \mathrm{pH}$ and Electrical conductivity $(\mathrm{ms} / \mathrm{cm})$ were measured in-situ using digital HANNA metre model HI 9813-5 between 8-11 GMT. Similarly the Dissolved Oxygen was measured in-situ using Digital DO meter model JPB-607A Power:6V.

\section{Bacteriological analysis}

Sterilization of Material Used: the materials used for this study were sterilized by appropriate technique. All glass wares such as Petri dishes, conical flasks, test tubes, beakers, McCartney bottles, etc., were thoroughly washed and sterilized in the hot air oven at $160^{\circ} \mathrm{C}$ for about 1 hour. The media used for these were Nutrient Agar (NA), MacConkey Agar (MA) and Eosin Methylene Blue (EMB). 28g, 48.5g, and $37.5 \mathrm{~g}$ of NA, MA and EMB were weighed and suspended in 1 litre of distilled water respectively. The mixture was then mixed properly and heated to dissolve the powder completely. After heating, the mouth of the flasks were plugged with cotton wool and wrapped with aluminium foil. The media were sterilized at $121^{\circ} \mathrm{C}$ for about $15 \mathrm{~min}$ in autoclave and then allowed to cool for about $45^{\circ} \mathrm{C}$ before pouring aseptically into the sterile Petri-dish.

Bacteriological procedures: serial dilution method was used for the enumeration of the bacterial count. $1 \mathrm{ml}$ of each of the samples were transferred into $9 \mathrm{ml}$ of sterile distilled water in the test tube. Serially, $1 \mathrm{ml}$ was taken from the $10^{-1}$ tube to another which makes $10^{-2}$. This was done up to $10^{-4}$ dilution. Then $1 \mathrm{~m}$ was taken from the $10^{-4}$ respectively into sterile Petri dishes and the molten agars that have been prepared earlier for bacterial growth were allowed to cool to about $45^{\circ} \mathrm{C}$ before pouring into each of the sterile Petri-dishes and allowed to solidify. After solidification, each of the plates were incubated at $37^{\circ} \mathrm{C}$ for the bacteria to grow between $24 \mathrm{hrs}-48 \mathrm{hrs}$. After the incubation period each of the plates were examined and counted using Bacteria Colony Counter in terms of colony forming units.

\section{Characterization and Identification of Bacterial Isolates}

The isolates were characterized $\mathrm{d}$ andientified after obtaining pure culture of isolates through repeated sub-culturing by using their Colonial morphology, Cellular morphology and Biochemical reaction and then identified by making reference to Bergey's manual of determinative bacteriology. Colonial and Cellular morphology; Gram staining; motility test; biochemical tests (including catalase, coagulase, oxidation, indole, Urease, Voges-Proskauer, methyl red, starch hydrolysis and sugar fermentation tests) were all conducted as described by Fawole and Oso (2007). 


\section{Data analysis}

The data were analyzed using IBM Statistical Package for Social Science (SPSS) version 20. All data are the mean of three replicates. The mean and standard deviation of each parameter were determined. The mean data were compared using one way Analysis of variance (ANOVA) and separated using Duncan Multiple Range Test (DMRT) at $\alpha=0.05$.

\section{Results}

This study helps to determine some ecological parameters and the bacteriological properties of fish pond water of two different kinds of rearing facilities (earthen ponds and concrete tanks). Earthen pond and concrete tank water samples were collected from different fish farms in the study area at the different sampling time (3days interval three times each).

\section{Physicochemical parameters of Earthen ponds and concrete tanks of selected farms}

Table 1a shows the physicochemical properties of the earthen pond water from the selected farms with DO range of $5.93 \mathrm{mg} / 1-6.43 \mathrm{mg} / 1$, TDS range of $243 \mathrm{mg} . / 1-451 \mathrm{mg} / 1$, $\mathrm{pH}$ range of $6.47-7.47$, Electrical conductivity range of $0.34 \mathrm{mS} / \mathrm{cm}-1.36 \mathrm{mS} / \mathrm{cm}$, Temperature range of $28.07^{\circ} \mathrm{C}-28.60^{\circ} \mathrm{C}$, Alkalinity range of $43.43 \mathrm{mg} / 1-53.17 \mathrm{mg} / 1$, Nitrate range of $2.11 \mathrm{mg} / 1-2.93 \mathrm{mg} / 1$ and Water Hardness range of $15.81 \mathrm{mg} / 1-26.25 \mathrm{mg} / \mathrm{l}$. Whereas Table $1 \mathrm{~b}$ shows the physicochemical properties of the concrete tank water from the selected farms with DO range of $6.30 \mathrm{mg} / 1-6.77 \mathrm{mg} / 1$, TDS range of $182.33 \mathrm{mg} / 1-306.67 \mathrm{mg} . / 1$, $\mathrm{pH}$ range of $6.80-8.27$, Electrical conductivity range of $0.25 \mathrm{mS} / \mathrm{cm}-1.62 \mathrm{mS} / \mathrm{cm}$, Temperature range of $28.10^{\circ} \mathrm{C}-28.90^{\circ} \mathrm{C}$, Alkalinity range of $48.40 \mathrm{mg} / 1-70.11 \mathrm{mg} / 1$, Nitrate range of $1.73 \mathrm{mg} / 1-2.18 \mathrm{mg} / 1$ and Water Hardness range of $20.44 \mathrm{mg} / 1-29.24 \mathrm{mg} / \mathrm{l}$.

Table 1a : Physicochemical characteristics of earthen pond water from selected farms

\begin{tabular}{lllllllll}
\hline Farms & \multicolumn{7}{l}{ Earthen ponds Physicochemical parameters } \\
\cline { 2 - 9 } & $\begin{array}{l}\text { DO } \\
(\mathbf{m g} / \mathbf{l})\end{array}$ & $\begin{array}{l}\text { TDS } \\
(\mathbf{m g} / \mathbf{l})\end{array}$ & $\mathbf{p H}$ & $\begin{array}{l}\mathbf{E C} \\
\mathbf{( m S / c m})\end{array}$ & $\begin{array}{l}\mathbf{T} \\
\left({ }^{\circ} \mathbf{C}\right)\end{array}$ & $\begin{array}{l}\text { ALK } \\
(\mathbf{m g} / \mathbf{l})\end{array}$ & $\begin{array}{l}\text { NIT } \\
(\mathbf{m g} / \mathbf{l})\end{array}$ & $\begin{array}{l}\text { WH } \\
(\mathbf{m g} / \mathbf{l})\end{array}$ \\
$\mathbf{A}$ & $6.17^{\mathrm{b}} \pm 0.06$ & $243.33^{\mathrm{a}} \pm 0.58$ & $6.83^{\mathrm{b}} \pm 0.06$ & $0.34^{\mathrm{b}} \pm 0.00$ & $28.60^{\mathrm{b}} \pm 0.00$ & $53.17^{\mathrm{e}} \pm 0.15$ & $2.12^{\mathrm{a}} \pm 0.01$ & $19.53^{\mathrm{b}} \pm 0.03$ \\
$\mathbf{B}$ & $5.93^{\mathrm{a}} \pm 0.06$ & $322.67^{\mathrm{a}} \pm 0.58$ & $7.47^{\mathrm{c}} \pm 0.06$ & $0.45^{\mathrm{c}} \pm 0.00$ & $28.43^{\mathrm{a}} \pm 0.06$ & $44.07^{\mathrm{b}} \pm 0.06$ & $2.43^{\mathrm{b}} \pm 0.01$ & $26.25^{\mathrm{c}} \pm 0.01$ \\
$\mathbf{C}$ & $6.43^{\mathrm{c}} \pm 0.11$ & $451.00^{\mathrm{e}} \pm 5.29$ & $6.47^{\mathrm{a}} \pm 0.15$ & $0.62^{\mathrm{d}} \pm 0.01$ & $28.07^{\mathrm{a}} \pm 0.06$ & $43.43^{\mathrm{a}} \pm 0.32$ & $2.11^{\mathrm{a}} \pm 0.01$ & $16.42^{\mathrm{a}} \pm 0.01$ \\
$\mathbf{D}$ & $6.10^{\mathrm{b}} \pm 0.10$ & $265.33^{\mathrm{b}} \pm 1.16$ & $7.33^{\mathrm{c}} \pm 0.06$ & $1.36^{\mathrm{e}} \pm 0.04$ & $28.27^{\mathrm{a}} \pm 0.55$ & $45.17^{\mathrm{c}} \pm 0.06$ & $2.93^{\mathrm{d}} \pm 0.01$ & $16.47^{\mathrm{a}} \pm 0.01$ \\
$\mathbf{E}$ & $6.23^{\mathrm{b}} \pm 0.06$ & $281.61^{\mathrm{c}} \pm 1.16$ & $6.63^{\mathrm{a}} \pm 0.15$ & $0.14^{\mathrm{a}} \pm 0.02$ & $29.87^{\mathrm{b}} \pm 0.70$ & $46.37^{\mathrm{d}} \pm 0.15$ & $2.73^{\mathrm{c}} \pm 0.01$ & $15.81^{\mathrm{a}} \pm 0.96$ \\
\hline
\end{tabular}

Table 1b: Physicochemical parameters of Concrete tanks water of selected farms

Farms Concrete tanks physicochemical parameters

\begin{tabular}{llllllll}
\hline $\begin{array}{l}\text { DO } \\
(\mathbf{m g} / \mathbf{l})\end{array}$ & $\begin{array}{l}\text { TDS } \\
(\mathbf{m g} / \mathbf{l})\end{array}$ & $\mathbf{p H}$ & $\begin{array}{l}\text { EC } \\
(\mathbf{m S} / \mathbf{c m})\end{array}$ & $\begin{array}{l}\text { TEM } \\
\left({ }^{\circ} \mathbf{C}\right)\end{array}$ & $\begin{array}{l}\text { ALK } \\
(\mathbf{m g} / \mathbf{l})\end{array}$ & $\begin{array}{l}\text { NIT } \\
(\mathbf{m g} / \mathbf{l})\end{array}$ & $\begin{array}{l}\text { WH } \\
(\mathbf{m g} / \mathbf{l})\end{array}$ \\
$6.43^{\mathrm{a}} \pm 0.06$ & $218.33^{\mathrm{a}} \pm 3.79$ & $7.70^{\mathrm{b}} \pm 0.10$ & $0.25^{\mathrm{a}} \pm 0.01$ & $28.60^{\mathrm{c}} \pm 0.00$ & $59.37^{\mathrm{c}} \pm 0.06$ & $1.73^{\mathrm{a}} \pm 0.29$ & $22.17^{\mathrm{c}} \pm 0.12$
\end{tabular}
$\mathbf{A}$
$6.43^{\mathrm{a}} \pm 0.06$
$218.33^{\mathrm{a}} \pm 3.79$
$7.70 \pm 0.10$
(1).25 \pm 0.01
$28.60 \pm 0.00$

$59.37 \pm 0.06$




\begin{tabular}{lllllllll} 
B & $6.30^{\mathrm{a}} \pm 0.00$ & $306.67^{\mathrm{e}} \pm 0.58$ & $6.80^{\mathrm{a}} \pm 0.00$ & $0.43^{\mathrm{d}} \pm 0.00$ & $28.10^{\mathrm{a}} \pm 0.10$ & $48.83^{\mathrm{b}} \pm 0.23$ & $1.73^{\mathrm{a}} \pm 0.06$ & $29.24^{\mathrm{d}} \pm 0.09$ \\
C & $6.67^{\mathrm{b}} \pm 0.06$ & $231.33^{\mathrm{d}} \pm 0.58$ & $7.70^{\mathrm{b}} \pm 0.10$ & $0.32^{\mathrm{c}} \pm 0.00$ & $28.37^{\mathrm{b}} \pm 0.06$ & $48.40^{\mathrm{a}} \pm 0.20$ & $1.77^{\mathrm{a}} \pm 0.15$ & $20.44^{\mathrm{a}} \pm 0.01$ \\
D & $6.67^{\mathrm{b}} \pm 0.15$ & $219.67^{\mathrm{b}} \pm 1.53$ & $8.10^{\mathrm{c}} \pm 0.10$ & $1.62^{\mathrm{e}} \pm 0.00$ & $28.90^{\mathrm{d}} \pm 0.00$ & $68.43^{\mathrm{d}} \pm 0.02$ & $2.15^{\mathrm{b}} \pm 0.06$ & $22.13^{\mathrm{c}} \pm 0.01$ \\
E & $6.77^{\mathrm{b}} \pm 0.06$ & $221.33^{\mathrm{c}} \pm 15.7$ & $8.27^{\mathrm{c}} \pm 0.23$ & $0.29^{\mathrm{b}} \pm 0.02$ & $28.17^{\mathrm{a}} \pm 0.12$ & $70.11^{\mathrm{e}} \pm 0.01$ & $2.18^{\mathrm{b}} \pm 0.06$ & $21.18^{\mathrm{b}} \pm 0.01$ \\
\hline
\end{tabular}

Table 1a \& 1b Note: mean followed by the same superscript within the same column are not significantly different at $\alpha=0.05$ based on Duncan Multiple Range Test (DMRT). DO (Dissolved oxygen); TDS (Total dissolved solids); EC (Electrical conductivity); T (Temperature), ALK (Alkalinity); NIT (Nitrate); WH (Water hardness).

\section{Bacteriological counts of earthen ponds and Concrete tanks in different sampling times}

Table 2 shows the total viable counts, total coliform counts and fecal coliform counts of the earthen pond water in the selected areas. The TVC, TCC and FCC of all the earthen pond water samples range from $5.10 \pm 0.12 \mathrm{LogCFU} / \mathrm{ml}$ to $5.83 \pm 0.04 \mathrm{LogCFU} / \mathrm{ml}, 4.46 \pm 0.15 \mathrm{LogCFU} / \mathrm{ml}$ to $5.48 \pm 0.03 \mathrm{LogCFU} / \mathrm{ml}$ and nil to $4.83 \pm 0.13 \mathrm{LogCFU} / \mathrm{ml}$ respectively. Moreover, in figure 1 ( $a, b$ and c) earthen pond water sampled from Farm C had the highest TVC 5.64 $\pm 0.10 \mathrm{LogCFU} / \mathrm{ml}$ while that of Farm D had the least TVC 5.26 $\pm 0.21 \mathrm{LogCFU} / \mathrm{ml}$. Similar is the case with TCC and FCC with TCC value of Farm C reaching $5.34 \pm 0.10 \mathrm{LogCFU} / \mathrm{ml}$ while that of Farm D had the least TCC 4.83 $\pm 0.41 \mathrm{LogCFU} / \mathrm{ml}$; and FCC value from Farm C recorded at 4.83 $\pm 0.13 \mathrm{LogCFU} / \mathrm{ml}$ and Farm D had least FCC $3.58 \pm 0.81 \mathrm{LogCFU} / \mathrm{ml}$. However, no FCC was recorded for Farm B. Furthermore, results recorded for bacteria counts in concrete ponds are presented in table 3. This shows variation with time of sampling. In table 3, the TVC, TCC and FCC of all the concrete tanks water samples ranged from $5.08 \pm 0.07 \mathrm{LogCFU} / \mathrm{ml}$ to $5.67 \pm 0.06 \mathrm{LogCFU} / \mathrm{ml} ; 4.26 \pm 0.24 \mathrm{LogCFu} / \mathrm{ml}$ to $5.36 \pm 0.26 \mathrm{LogCFU} / \mathrm{ml}$; and 0.00 to $4,77 \pm 0.07 \mathrm{LogCFU} / \mathrm{ml}$ respectively. And grossly, Figure 2 ( $\mathrm{a}, \mathrm{b}$ and c) shows that the concrete tank water sample from Farm C had the highest TVC 5.39 $\pm 0.14 \mathrm{LogCFU} / \mathrm{ml}$ while that of Farm B had the least TVC $5.17 \pm 0.25 \mathrm{LogCFU} / \mathrm{ml}$. Where Farm E recorded the highest TCC $5.23 \pm 0.11 \mathrm{LogCFU} / \mathrm{ml}$ while that of Farm B had the least TCC $4.81 \pm 0.48$ LogCFU $/ \mathrm{ml}$. Concrete water sampled from Farm E had the highest FCC 4.57 $\pm 0.11 \mathrm{Log}$ CFU $/ \mathrm{ml}$ while that of Farm A, Farm B and Farm C showed no value FCC counts.

Presented in table $4 \mathrm{a}$ and $4 \mathrm{~b}$ are the distribution of bacteria occurrence in farm waters in 3 different sampling occasions. Altogether, total 8 bacteria were isolated and their occurence showed variation between farms, culturing facilities as well as temporal variation. Finally, in table 5, the percentage occurrence of this bacteria group was estimated for the two exclosure systems. In the case of earthen pond waters, Staphylococcus spp and Enterobacter group showed highest occurence (20\%) while Proteus spp and Esherichia coli showed lowest percentage occurrence of 8\%. Similarly, in the concrete water sampled, Staphylococcus spp and Enterobacter group showed highest occurence approximately $22 \%$ and $18 \%$ respectively. While lower occurrence was attributed to Escherichia coli and Proteus spp (8\%), with lowest occurrence recorded for Pseudomonas spp (approx. 6\%).

Table 2: Bacteriological counts of Farm A-E earthen pond water of different sampling time

\begin{tabular}{lllll}
\hline & Sampling time & TVC $(\log \mathbf{c f u} / \mathbf{m l})$ & $\begin{array}{l}\text { TCC(Log } \\
\text { cfu/ml })\end{array}$ & FCC(Log cfu/ml) \\
Farm A & $1^{\text {st }}$ sampling & $5.41^{\mathrm{a}} \pm 0.08$ & $5.04^{\mathrm{a}} \pm 0.07$ & $4.54^{\mathrm{b}} \pm 0.28$ \\
& $2^{\text {nd }}$ sampling & $5.61^{\mathrm{a}} \pm 0.15$ & $5.28^{\mathrm{b}} \pm 0.05$ & $2.77^{\mathrm{ab}} \pm 2.40$ \\
& $3^{\text {rd }}$ sampling & $5.83^{\mathrm{b}} \pm 0.04$ & $5.48^{\mathrm{c}} \pm 0.03$ & $0.00^{\mathrm{a}} \pm 0.00$ \\
\cline { 2 - 5 } Farm B & $1^{\text {st }}$ sampling & $5.11^{\mathrm{a}} \pm 0.10$ & $4.83^{\mathrm{a}} \pm 0.16$ & $0.00 \pm 0.00$ \\
& $2^{\text {nd }}$ sampling & $5.46^{\mathrm{b}} \pm 0.13$ & $5.03^{\mathrm{ab}} \pm 0.12$ & $0.00 \pm 0.00$
\end{tabular}




\begin{tabular}{lllll} 
& $3^{\text {rd }}$ sampling & $5.52^{\mathrm{b}} \pm 0.13$ & $5.14^{\mathrm{b}} \pm 0.10$ & $0.00 \pm 0.00$ \\
\cline { 2 - 5 } Farm C & $1^{\text {st }}$ sampling & $5.56^{\mathrm{a}} \pm 0.14$ & $5.25^{\mathrm{a}} \pm 0.11$ & $0.00^{\mathrm{a}} \pm 0.00$ \\
& $2^{\text {nd }}$ sampling & $5.61^{\mathrm{a}} \pm 0.09$ & $5.33^{\mathrm{a}} \pm 0.14$ & $4.83^{\mathrm{b}} \pm 0.13$ \\
& $3^{\text {rd }}$ sampling & $5.76^{\mathrm{a}} \pm 0.09$ & $5.44^{\mathrm{a}} \pm 0.12$ & $0.00^{\mathrm{a}} \pm 0.00$ \\
\cline { 2 - 5 } Farm D & $1^{\text {st }}$ sampling & $5.10^{\mathrm{a}} \pm 0.12$ & $4.46^{\mathrm{a}} \pm 0.15$ & $4.39^{\mathrm{b}} \pm 0.36$ \\
& $2^{\text {nd }}$ sampling & $5.17^{\mathrm{a}} \pm 0.09$ & $4.75^{\mathrm{a}} \pm 0.18$ & $0.00^{\mathrm{a}} \pm 0.00$ \\
& $3^{\text {rd }}$ sampling & $5.50^{\mathrm{a}} \pm 0.09$ & $5.27^{\mathrm{b}} \pm 0.14$ & $2.77^{\mathrm{a} b} \pm 2.40$ \\
\cline { 2 - 5 } Farm E & $1^{\text {st }}$ sampling & $5.26^{\mathrm{a}} \pm 0.15$ & $4.59^{\mathrm{a}} \pm 0.11$ & $0.00^{\mathrm{a}} \pm 0.00$ \\
& $2^{\text {nd }}$ sampling & $5.41^{\mathrm{a}} \pm 0.07$ & $4.97^{\mathrm{b}} \pm 0.14$ & $4.26^{\mathrm{b}} \pm 0.24$ \\
& $3^{\text {rd }}$ sampling & $5.62^{\mathrm{b}} \pm 0.07$ & $5.38^{\mathrm{c}} \pm 0.06$ & $0.00^{\mathrm{a}} \pm 0.00$ \\
\hline
\end{tabular}

Means with the same superscript in columns are not significantly different at $\alpha=0.05$ based on the Duncan Multiple Range Test (DMRT). TVC (Total Viable Count); TCC (Total Coliform Count); FCC (Fecal Coliform Count) Note: Values presented as mean \pm standard deviation of triplicates.

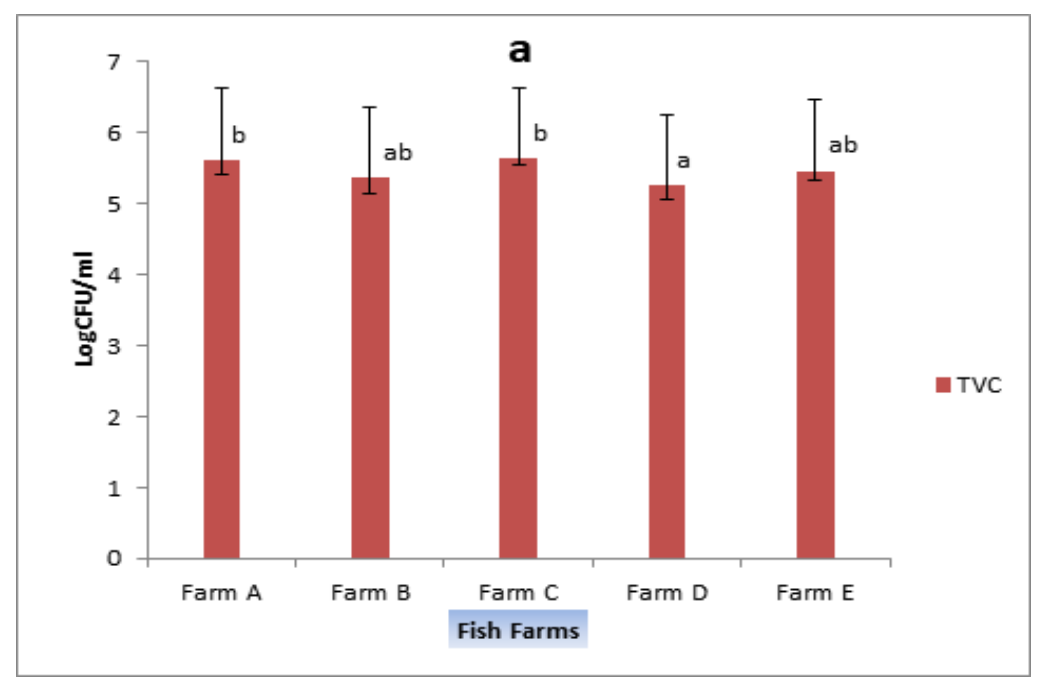



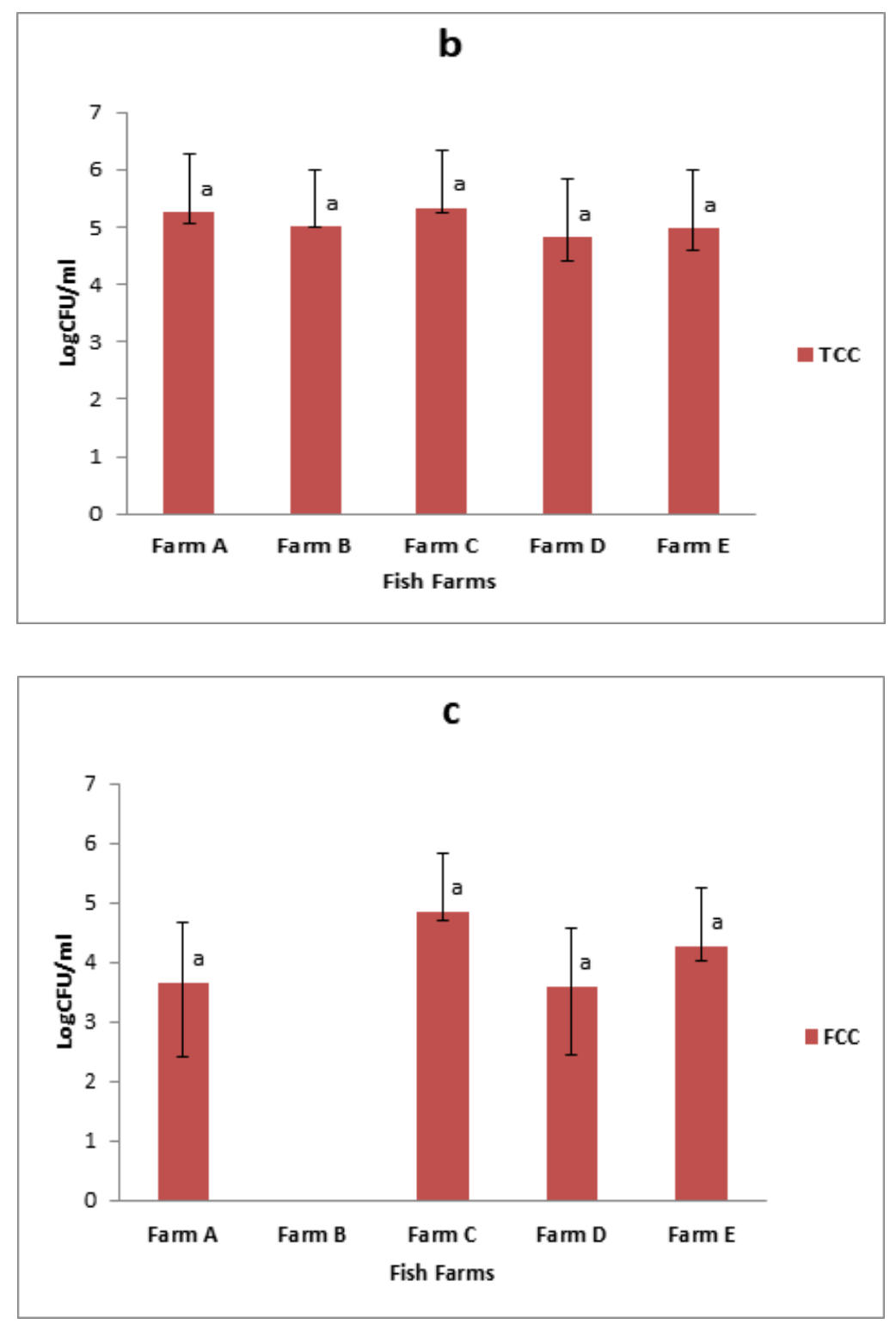

Figure 1a,b and c: gross TVC, TCC and FCC in earthen pond water of fish farm. Bars with the same alphabets are not significantly different at $\alpha=0.05$ based on the Duncan Multiple Range Test (DMRT). Notes: Farms not barred shows no recorded value; Values presented as mean \pm standard deviation of replicates.TVC (Total Viable Count); TCC (Total Coliform Count); FCC (Fecal Coliform Count).

\section{Bacteriological counts of Farm A-E Concrete tank waters in different sampling time}

Table 3: Bacteriological counts of Farm A-E concrete tank water of different sampling time

\begin{tabular}{lllll}
\hline & Sampling time & TVC $(\log$ cfu $/ \mathbf{m l})$ & $\begin{array}{l}\text { TCC(Log } \\
\text { cfu/ml })\end{array}$ & FCC(Log cfu/ml) \\
Farm A & $1^{\text {st }}$ sampling & $5.16^{\mathrm{a}} \pm 0.15$ & $4.88^{\mathrm{a}} \pm 0.16$ & $0.00 \pm 0.00$ \\
& $2^{\text {nd }}$ sampling & $5.11^{\mathrm{a}} \pm 0.07$ & $4.97^{\mathrm{a}} \pm 0.20$ & $0.00 \pm 0.00$
\end{tabular}




\begin{tabular}{lllll} 
& $3^{\text {rd }}$ sampling & $5.67^{\mathrm{b}} \pm 0.06$ & $5.36^{\mathrm{b}} \pm 0.26$ & $0.00 \pm 0.00$ \\
\cline { 2 - 5 } Farm B & $1^{\text {st }}$ sampling & $5.46^{\mathrm{a}} \pm 0.09$ & $5.19^{\mathrm{a}} \pm 0.10$ & $0.00 \pm 0.00$ \\
& $2^{\text {nd }}$ sampling & $4.99^{\mathrm{a}} \pm 0.14$ & $4.26^{\mathrm{a}} \pm 0.24$ & $0.00 \pm 0.00$ \\
Farm C & $3^{\text {rd }}$ sampling & $5.09^{\mathrm{b}} \pm 0.13$ & $4.99^{\mathrm{b}} \pm 0.14$ & $0.00 \pm 0.00$ \\
\cline { 2 - 5 } & $1^{\text {st }}$ sampling & $5.23^{\mathrm{a}} \pm 0.08$ & $4.92^{\mathrm{a}} \pm 0.21$ & $0.00 \pm 0.00$ \\
& $2^{\text {nd }}$ sampling & $5.44^{\mathrm{b}} \pm 0.06$ & $5.20^{\mathrm{ab}} \pm 0.10$ & $0.00 \pm 0.00$ \\
Farm D & $3^{\text {rd }}$ sampling & $5.50^{\mathrm{b}} \pm 0.04$ & $5.29^{\mathrm{b}} \pm 0.11$ & $0.00 \pm 0.00$ \\
\cline { 2 - 5 } & $1^{\text {st }}$ sampling & $5.17^{\mathrm{ab}} \pm 0.06$ & $5.03^{\mathrm{b}} \pm 0.12$ & $4.77^{\mathrm{b}} \pm 0.07$ \\
& $2^{\text {nd }}$ sampling & $5.08^{\mathrm{a}} \pm 0.07$ & $4.63^{\mathrm{a}} \pm 0.31$ & $4.00^{\mathrm{a}} \pm 0.00$ \\
& $3^{\text {rd }}$ sampling & $5.25^{\mathrm{b}} \pm 0.07$ & $5.08^{\mathrm{b}} \pm 0.07$ & $4.26^{\mathrm{a}} \pm 0.24$ \\
\cline { 2 - 5 } Farm E & $1^{\text {st }}$ sampling & $5.22^{\mathrm{a}} \pm 0.10$ & $5.11^{\mathrm{a}} \pm 0.10$ & $0.00^{\mathrm{a}} \pm 0.00$ \\
& $2^{\text {nd }}$ sampling & $5.29^{\mathrm{a}} \pm 0.10$ & $5.27^{\mathrm{ab}} \pm 0.11$ & $4.46^{\mathrm{b}} \pm 0.15$ \\
& $3^{\text {rd }}$ sampling & $5.53^{\mathrm{b}} \pm 0.06$ & $5.32^{\mathrm{b}} \pm 0.07$ & $4.67^{\mathrm{b}} \pm 0.19$ \\
\hline
\end{tabular}

Means with the same superscript in columns are not significantly different at $\alpha=0.05$ based on the Duncan Multiple Range Test (DMRT). TVC (Total Viable Count); TCC (Total Coliform Count); FCC (Fecal Coliform Count) Note: Values presented as mean \pm standard deviation of triplicates.

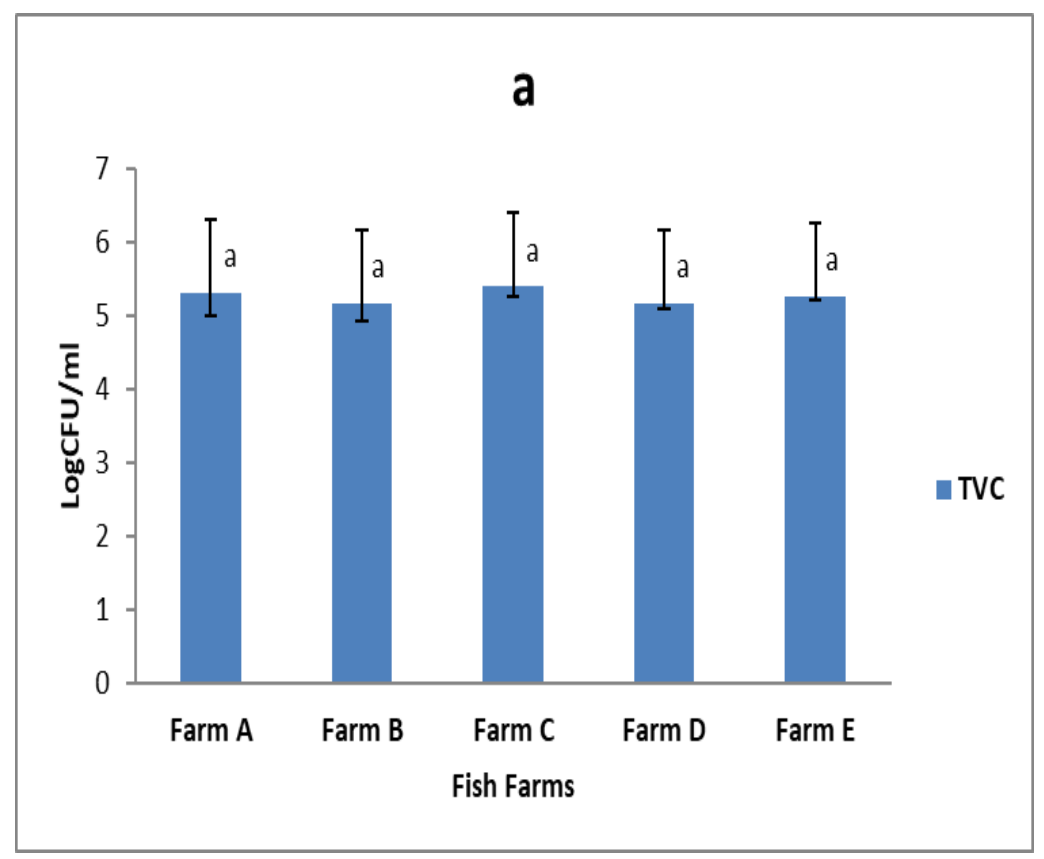



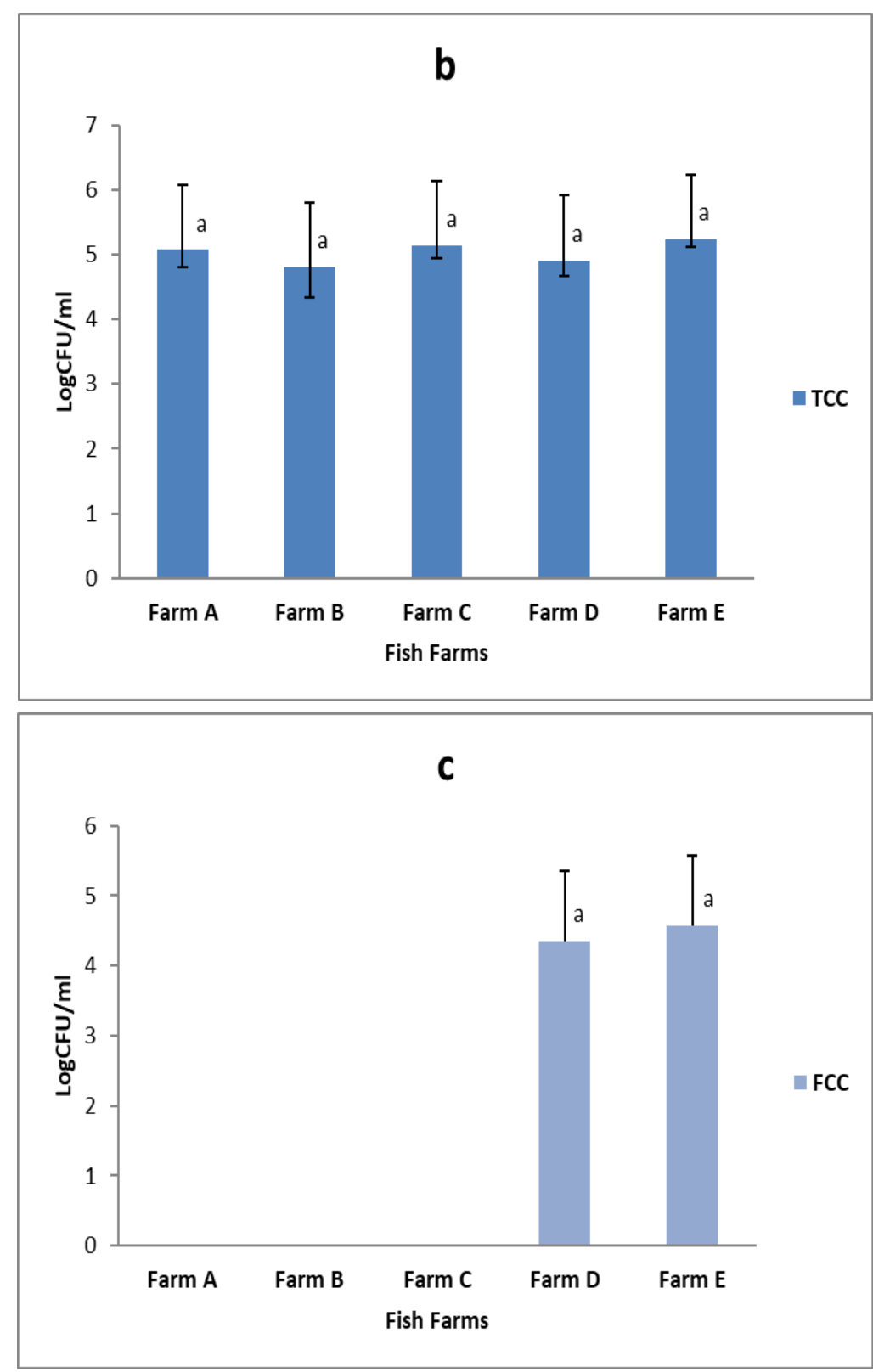

Figure 2a,b and c: gross TVC, TCC and FCC in concrete tank water of fish farm. Bars with the same alphabets are not significantly different at $\alpha=0.05$ based on the Duncan Multiple Range Test (DMRT). Notes: Farms not barred shows no recorded value; Values presented as mean \pm standard deviation of replicates.TVC (Total Viable Count); TCC (Total Coliform Count); FCC (Fecal Coliform Count).

Table 4a: Distribution of occurrence of Bacterial species of earthen pond water

\begin{tabular}{|c|c|c|c|c|c|c|c|c|c|c|}
\hline \multirow[t]{2}{*}{ Bacterial isolates } & \multicolumn{3}{|c|}{$\begin{array}{l}\text { Farm A earthen } \\
\text { pond water }\end{array}$} & \multicolumn{3}{|c|}{$\begin{array}{l}\text { Farm B } \\
\text { earthen pond } \\
\text { water }\end{array}$} & \multicolumn{2}{|c|}{$\begin{array}{l}\text { Farm } C \text { earthen } \\
\text { pond water }\end{array}$} & $\begin{array}{l}\text { Farm D } \\
\text { earthen pond } \\
\text { water }\end{array}$ & $\begin{array}{l}\text { Farm E } \\
\text { earthen } \\
\text { pond water }\end{array}$ \\
\hline & $1^{\text {st }}$ & $2^{\text {nd }}$ & $3^{\text {rd }}$ & $1^{\text {st }}$ & $2^{\text {nd }}$ & $3^{\text {rd }}$ & $1^{\text {st }} \quad 2^{\text {nd }}$ & $3^{\text {rd }}$ & $1^{\text {st }} 2^{\text {nd }} 3^{\text {rd }}$ & $1^{\text {st }} 2^{\text {nd }} 3^{\text {rd }}$ \\
\hline
\end{tabular}


Streptococcus spp

Proteus spp

Enterobacter spp

Serratia spp

Bacillus spp

Escherichia coli

Pseudomonas spp

Key: (+) presence of bacteria isolate, (-) absence of bacteria isolates

Table 4a: Distribution of occurrence of Bacterial species of earthen pond water

\begin{tabular}{|c|c|c|c|c|c|c|c|c|c|c|c|c|c|c|c|}
\hline \multirow[t]{2}{*}{ Bacterial isolates } & \multicolumn{3}{|c|}{$\begin{array}{l}\text { Farm A } \\
\text { concrete tank } \\
\text { water }\end{array}$} & \multicolumn{3}{|c|}{$\begin{array}{l}\text { Farm B } \\
\text { concrete } \\
\text { tank water }\end{array}$} & \multicolumn{3}{|c|}{$\begin{array}{l}\text { Farm C } \\
\text { concrete } \\
\text { tank water }\end{array}$} & \multicolumn{3}{|c|}{$\begin{array}{l}\text { Farm D } \\
\text { concrete } \\
\text { tank water }\end{array}$} & \multicolumn{3}{|c|}{$\begin{array}{l}\text { Farm E } \\
\text { concrete } \\
\text { tank water }\end{array}$} \\
\hline & $1^{\text {st }}$ & $2^{\text {nd }}$ & $3^{\text {rd }}$ & $1^{\mathrm{s}}$ & $2^{\text {nd }}$ & $3^{\text {rd }}$ & $1^{\text {st }}$ & $2^{\text {nd }}$ & $3^{\text {rd }}$ & $1^{\text {st }}$ & $2^{\text {nd }}$ & $3^{\text {rd }}$ & $1^{\text {st }}$ & $2^{\text {nd }}$ & $3^{\text {rd }}$ \\
\hline Staphylococcus spp & + & + & + & + & - & - & + & + & + & + & + & - & + & + & + \\
\hline Streptococcus spp & - & + & + & + & + & + & + & + & + & + & + & + & - & - & - \\
\hline Proteus spp & - & + & - & - & + & + & - & - & + & - & - & - & - & - & + \\
\hline Enterobacter spp & + & + & + & + & + & + & + & + & + & + & + & + & + & + & + \\
\hline Serratia spp & + & - & + & + & - & - & + & - & - & - & + & - & - & - & - \\
\hline Bacillus spp & + & + & - & - & + & + & + & + & + & + & + & - & - & - & + \\
\hline Escherichia coli & - & - & - & - & - & - & - & - & - & + & + & + & - & + & + \\
\hline Pseudomonas spp & + & + & - & - & + & - & - & - & - & - & - & - & - & - & - \\
\hline
\end{tabular}

Key: $(+)$ presence of bacteria isolate, $(-)$ absence of bacteria isolates

Table 5: Percentage occurrence of bacteria in earthen pond water

Bacteria
Earthen pond water Concrete tank (\%)

(\%) 


\begin{tabular}{lll}
\hline Staphylococcus spp & 20 & 18 \\
Streptococcus spp & 12 & 16 \\
Proteus spp & 8 & 8 \\
Enterobacter spp & 20 & 22 \\
Serratia spp & 16 & 8 \\
Bacillus spp & 9 & 15 \\
Escherichia coli & 8 & 8 \\
Pseudomonas spp & 7 & 6 \\
\hline
\end{tabular}

\section{Discussion}

The present study was designed to investigate the health status of aquaculture settings practicing stagnant water aquaculture. It is well established that water used for the culture of fish will not give maximum production if the 'physicochemical' parameters are not optimal for fish. And subsequently presenting fish to stressor(s) experience (Santos et al., 2010). One outcome of this is increased susceptibility to diseases (Fridell et al., 2007; Boyd, 2017). For example, channel catfish held in water with suboptimal quality (dissolved oxygen, carbon dioxide and ammonia) experienced reduced survival when injected intraperitoneally with sublethal doses of Aeromonas hydrophila conversely to fish kept in favourable water condition (Walters \& Plumb, 1980). In the present study area investigated, all the parameters observed (Table 1a \& b), both in earthen and concrete tank rearing enclosures, fall within the recommended range in the literature for aquaculture (e.g., Dalvi et al., 2009; Boyd, 2017). It is worth stating here that, the extremely reduced nitrate concentrations (Table $1 \mathrm{a} \& \mathrm{~b}$ ) recorded might be due to the increased utilisation by present bacteria (Schneider et al., 2005). This is a similar principle in conventional static water aquaculture systems, biofloc system (Poh, 2014) and aquamimicry (Romano, 2017; Romano and Kumar, 2017). Where the nitrogenous wastes including ammonia is converted to bacterial biomass (Schneider et al., 2005). This is especially beneficial for aquaculture performance. For example, it has been documented that freshwater fish reared at 0,10 , and $100 \mathrm{mg} / 1$ nitrate showed increased specific growth rate and food conversion efficiency relative to higher nitrate concentration (e.g., $>100-1000 \mathrm{mg} / \mathrm{l}$ ) (Monsees et al., 2017).

Essentially, following the investigation of microbial profile, the most obvious finding to emerge from bacteria analyses of different sampling time is the increased concentration of total viable and coliform bacteria counts (TVC and TCC, Table $2 \& 3$ ). Contrary to expectation, unlike earthen pond waters, this trend was not experienced in concrete tank waters (Table 3). A possible explanation for this might be the fact that the majority of farm operators using concrete water aquaculture replenish the water use due to inability of the concrete rearing environment to naturally process wastes relative to earth ponds (Jha \& Barat, 2005; Jha, 2019). Which otherwise could result in reduced performance of the stock (Das et al., 2021). Therefore, one associable consequence of increasing concentration over time is the possible increase in pathogenic challenges over time, probably due to accumulation of organic substrates. Because it has been reported that growth of pathogenic bacteria such as Escherichia coli, Vibrio cholerae and Pseudomonas aeruginosa is affected by the composition and concentration of organic carbon in the water (Vital et al., 2010). Collectively, the presence of Gram-negative bacteria provides indication of presence of pathogenic bacteria groups. Especially, the presence of Escherichia coli provides indication of presence of pathogens (Ampofo \& Clerk, 2010). Although not isolated from this study, Vibrio cholerae, a pathogenic bacteria, has been isolated in other regions from aquaculture rearing facilities (Njoku et al., 
2015) and has been implicated to account for several diarrhea cases in Nigeria. For example, in the 2017 incident in the Ilorin metropolis, the culture positive rate of the Vibrio cholerae isolates was 41\% (Amadu et al., 2021). Compared to the $78 \%$ level of occurrence reported by Borah et al. (2010), Escherichia coli found in the present study was lower $(8 \%)$ in both rearing enclosures and the regular trend recorded for other bacteria groups (TVC and TCC) was not witnessed for the fecal coliform group (Table $2 \& 3$ ). It seems appropriate to associate this irregularity recorded to the long time exposure to solar radiation (Chandran \& Hatha, 2003) due to minimal water exchange. Because long term exposure to UV radiation has been reportedly claimed to inactivate $E$. coli cells (Muela et al., 2000). In fact, up to $90 \%$ of E.coli cells have been reported to be disrupted by sunlight exposure (Chandran \& Hatha, 2003).

Before proceeding to examining the favourability of isolated bacteria, it is important to mention that during the period of study, no gross sign of disorders were observed. In another study, increased tolerance of the culture species, Clarias gariepinus, has been reasonably attributed (Oni et al., 2013). Furthermore, other isolated bacteria including Bacillus spp and Staphylococcus spp form beneficial components of the pond ecosystem. And due to constant contact and inevitable ingestion of surrounding water, farmed fish are strongly influenced by the microorganisms contained in the water (Defoirdt et al., 2011; El-Saadony et al., 2021). For example, previous research has established that there is similarity between intestinal and culture water microbiota (Wu et al., 2012). In the study area investigated, of the bacteria group isolated, Staphylococcus spp appeared to show the highest occurrence (up to 18-20\%). This gives an indication that cultured stock benefits, on one hand, from the antimicrobial effects of these bacteria (Sumi et al., 2014; Santos et al., 2018) and, on the other hand, reduced food conversion ratio (FCR). For instance, using Bacillus $s p$ and photosynthetic bacteria isolated from carp ponds as probiotics, Yanbo \& Zirong (2006) reported a significant increase in growth performance and FCR. However, a note of caution is due here, due to the static water aquaculture practice in the study area, there is risk of opportunistic infection, as a result of overwhelming growth of bacteria or prolonged stimulation time of immune response (Cruz et al., 2012).

Conclusively, there is no doubt the open nature of aquatic environments present limitations to total exclusion of pathogens. As presented in this study, the appearance of Escherichia coli is an indication of possible presence of pathogens that may grow overwhelmingly to suppress the immune system of aquaculture stock (Hess et al., 2021), with potential consequences of disease outbreak. Arguably, compared to other livestock zoonosis, there has not been enough evidence examining the relationships between aquaculture originated pathogens and workers health threats, however, possible contamination of aquaculture products has been evaluated. For example, it was reported that tissue of fish cultured in fertilised ponds showed Salmonella contamination compared to unfertilised ponds (Ampofo \& Clerk, 2010). This is an area requiring attention especially with mounting concern 'one health initiatives' requirements. Nevertheless, from environmentalists' perspective, the practice of static water aquaculture is an approach that limits the use of freshwater and frequent interactions between aquaculture and the wild environment. This has the advantage of limiting pathogenic interactions between these artificial and natural ecosystems. Yet, there is the risk of increased pathogenicity due to continuum in pathogenic growth. Therefore it may be appropriate to recommend that aquaculture practicing zero water exchange should adapt principles from biofloc technology or aquamimicry. The latter concept being a conventional zero water exchange aquaculture, concurrently provides the advantages of increasing growth of heterotrophic organisms, maximises biosecurity, and accumulation of organics. Interestingly, a study comparing culture conditions. Biofloc systems and stagnant water renewal reported a greater performance in a biofloc system supplemented with probiotics (Mohammadi et al., 2021). 


\section{References}

Akankali, J.A., Abowei, J.F.N. \& Eli, A. (2011). "Pond fish culture practices in Nigeria". Advanced Journal of Food Science and Technology. 181-195.

Alaliyat, S., Yndestad, H. and Davidsen, P.I. (2019). An agent-based approach for predicting patterns of pathogen transmission between aquaculture sites in the Norwegian fjords. Aquaculture, 505: 98-111.

Alday de Graindorge, V., and Griffith, D. (2001). Ecuador: national review on management strategies for major diseases in shrimp aquaculture. p17-19. In: Subasinghe, R., Arthur, R., Phillips, M.J., Reantaso, M. (Eds.). WB/NACA/WWF/FAO 2001. "Thematic Review on Management Strategies for Major Diseases in Shrimp Aquaculture" Proceedings of a Workshop held in Cebu, Philippines on 28-30 November 1999. Edited by R. Subasinghe, R. Arthur, M. J. Phillips and M. Reantaso.The World Bank (WB), Network of Aquaculture Centres in Asia-Pacific (NACA), World Wildlife Fund (WWF) and Food and Agriculture Organization of the United Nations (FAO) Consortium Program on Shrimp Farming and the Environment. Work in Progress for Public Discussion. Published by the Consortium. 135pages. file:///C:/Users/USER/Downloads/1485335932_thematic-review-on-management-strategies-for-major-dise ases-in-shrimp-aquaculture.pdf.

Amadu, D. O., Abdullahi, I. N., Seibu, E., Fadeyi, A., Kamaldeen, K., Akanbi, A. A., Okwume, C. C., Amadu, M. B., \& Nwabuisi, C. (2021). Retrospective Analysis of the Serovars and Antibiogram of Vibrio cholerae Isolates of the 2017 Ilorin Cholera Outbreak, Nigeria. Infection \& chemotherapy. 53: 368-373.

Ampofo, J.A. \& Clerk, G.C. (2010). Diversity of Bacteria contaminants in Tissues of fish cultured in organic waste fertilized ponds; Health implications. The open fish science Journal 3:142 - 146.

Arias-Moscoso, J.L., Espinoza-Barrón, L.G., Miranda-Baeza, A., Rivas-Vega, M.E. \& Nieves-Soto, M. (2018). Effect of commercial probiotics addition in a biofloc shrimp farm during the nursery phase in zero water exchange. Aquaculture Reports. 11: 47-52.

Borah, M., Dutta, J. \& Misra, A.K. (2010). The bacteriological quality of drinking water in Golaghat Sub-division of Golaghat district, Assam, India. International Journal of Chemical Technological Research. 2: 1843-1851.

Boyd, C.E. (2017). Chapter 6 - General Relationship Between Water Quality and Aquaculture Performance in Ponds, Editor(s): Galina Jeney, Fish Diseases, Academic Press. 147-166.

Chandran, A. \& Hatha, A. M. (2003). Survival of Escherichia coli in a tropical estuary. The South Pacific Journal of Natural and Applied Sciences. 21: 41-46.

Cruz, P.M., Iba'nez, A.L. Hermosillo, O.A.M. \& Saad, H.C.R. (2012). Use of probiotics in Aquaculture. International Scholarly Research Network Microbiology. 2012. 13pp.

Dalvi, R.S. Pal, A.K., Tiwari, L.R., Das, T. \& Baruah, K. (2009). Thermal tolerance and oxygen consumption rates of the catfish Horabagrus brachysoma (Günther) acclimated to different temperatures. Aquaculture. 295:116-119.

Das, P.C., Kamble, S.P., Sahoo, N. \& Velmurugan. P. (2021).Influence of water exchange rates on fingerling production in Indian major carps in large outdoor concrete tanks. Aquacultural Engineering. 95: 102203.

Defoirdt, T., Thanh, L.D., Van Delsen, B., De Schryver, P., Sorgeloos, P., Boon, N. \& Bossier, P. (2011). $\mathrm{N}$-acylhomoserine lactone-degrading Bacillus strains isolated from aquaculture animals. Aquaculture. 311: 258-260.

Dekić, S., Klobučar, G., Ivanković, T., Zanella, D., Vucić, M., Bourdineaud, J. \& Hrenović, J. (2018). Emerging human pathogen Acinetobacter baumannii in the natural aquatic environment: a public health risk?, International Journal of Environmental Health Research. 28:(3) 315-322. 
Eissa, N. \& Wang, H.P. (2016), Transcriptional stress responses to environmental and husbandry stressors in aquaculture species. Review in Aquaculture. 8: 61-88.

El-Saadony, M.T., Alagawany, M., Patra, A.K., Kar, I., Tiwari, R., Dawood, M.A.O., Dhama, K. \& Abdel-Latif, H.M.R. (2021). The functionality of probiotics in aquaculture: An overview. Fish \& Shellfish Immunology. 117:36-52.

Emmanuel, O., Chinenye, A., Oluwatobi, A. \& Peter, K. (2014). Review of Aquaculture Production and Management in Nigeria. American Journal of Experimental Agriculture. 4(10): 1137-1151.

Fakorede, C.N., Fatokun, E.N., Philip K.B., Iwu, C.J. Jaja, I.F. (2019). Bacteriological Assessment and Antibiotics Susceptibility Profile of Bacteria Recovered from Pond Water, Fish Skin, and Gut in Ile-Ife, Osun State, Nigeria. Preprints.

Fawole, M.O. and Oso, B.A. (2007). Laboratory Manual of Microbiology. Spectrum Books Limited, Ibadan, 123 pp.

Fridell, F. Gadan, K. Sundh,H. Taranger, G.L. Glette, J. Olsen, R.E. Sundell, K. Evensen, Ø. (2007). Effect of hyperoxygenation and low water flow on the primary stress response and susceptibility of Atlantic salmon Salmo salar L. to experimental challenge with IPN virus. Aquaculture. 270: 23-35.

Georgiades, E., Fraser, R. \& Jones, B. (2016). Options to strengthen On-farm biosecurity management for commercial and Non-commercial aquaculture. Aquaculture Unit. Technical Paper No: 2016/47. Available at: https://researchrepository.murdoch.edu.au/id/eprint/42722/1/on-farm-aquaculture-biosecurity.pdf.

Hasimuna, O.J., Maulu, S. \& Mphande, J. (2020). Aquaculture Health Management Practices in Zambia: Status, Challenges and Proposed Biosecurity Measures. Journal of Aquaculture Research and Development. 11: $1-6$.

Hess, A., Baum, C., Schiessl, K., Besmer, M.D., Hammes, F. \& Morgenroth, E. (2021). Stagnation leads to short-term fluctuations in the effluent water quality of biofilters: A problem for greywater reuse? Water Research X. 13: 100120 .

Islam, M., Drasar, B., \& Sack, R. (1993). The Aquatic Environment as a Reservoir of Vibrio cholerae: A review. Journal of Diarrhoeal Diseases Research, 11(4): 197-206.

Jha, P. \& Barat, S. (2005). Effect of water exchange on water quality and the production of ornamental carp (Cyprinus carpio var. Koi L.) cultured in concrete tanks manured with poultry excreta. Archive of Polish Fisheries. 13: 77-90.

Jha, P. (2019). Evaluation of different water exchange regimes for optimizing growth and production of koi carp, Cyprinus carpio in tanks. Iranian Journal of Ichthyology. 6: 283-291.

Lightner, D.V. Redman, R.M. Pantoja, C.R. Tang, K.F.J. Noble, B.L.Schofield, P. Mohney, L.L. Nunan, L.M. Navarro, S.A. (2012). Historic emergence, impact and current status of shrimp pathogens in the Americas. Journal of Invertebrate Pathology. 110: 174-183.

Mohammadi, G., Rafiee, G., Tavabe, K.R., Abdel-Latif, H.M.R. \& Dawood, M.A.O. (2021).The enrichment of diet with beneficial bacteria (single- or multi- strain) in the biofloc system enhanced the water quality, growth performance, immune responses, and disease resistance of Nile tilapia (Oreochromis niloticus). Aquaculture. 539:736640.

Monsees, H., Klatt, L., Kloas, W. \& Wuertz, S. (2017). Chronic exposure to nitrate significantly reduces growth and affects the health status of juvenile Nile tilapia (Oreochromis niloticus L.) in recirculating aquaculture systems. Aquaculture Research. 48: 3482-3492.

Muela, A., García-Bringas, J., Arana, I. \& Barcina, I. (2000). The Effect of Simulated Solar Radiation on Escherichia coli: The Relative Roles of UV-B, UV-A, and Photosynthetically Active Radiation. Microbial Ecology. 39:65-71. 
Njoku, O.E., Agwa, O.K. \& Ibiene, A.A., (2015). An investigation of the microbiological and physicochemical profile of some fish pond water within the Niger Delta region of Nigeria. African journal offood Science. 9:155-162.

Nzeh, C.G. \& Udeze, A.O. (2012). Microbial survey of fish ponds and mineral composition of Clarias gariepinus in Ilorin metropolis. International Journal of Biology and Chemical Sciences. 6(2): 832 - 837.

Oni, T.A., Olaleye, V.E. \& Omafuvbe, B.O. (2013). Preliminary studies on associated bacterial and fungal load of artificially cultured Clarias gariepinus, Burchell 1822 fingerlings. Ife Journal of Science. 15: 9 - 16.

Poh, Y.T. (2014). Use of biofloc in shrimp farming. AQUA Culture Asia Pacific Magazine. Pp. 13-16.

Romano, N. \& Kumar, V. (2017). Vegetarian shrimp: pellet-free shrimp farming. World Aquaculture. 37: 36-38.

Romano, N. (2017). Aquamimicry: A revolutionary concept for shrimp farming. The Global Aquaculture Advocate. Based on attendance at Aquamimicry workshop in Thailand. Organised by the Aquamimicry Aquaculture Alliance. 5pp.

Santos, G.A., Schrama, J.W., Mamauag, R.E.P.. Rombout, J.H.W.M. \& Verreth. J.A.J. (2010). Chronic stress impairs performance, energy metabolism and welfare indicators in European seabass (Dicentrarchus labrax): The combined effects of fish crowding and water quality deterioration. Aquaculture. 299:73-80

Santos, R.A., Oliva-Teles, A., Saavedra, M.J., Enes, P. \& Serra, C.R. (2018). Bacillus spp. as source of Natural Antimicrobial Compounds to control aquaculture bacterial fish pathogens. Front. Mar. Sci. Conference Abstract: IMMR'18 | International Meeting on Marine Research 2018. Available at: https://www.researchgate.net/profile/Aires-Oliva-Teles/publication/328388972_Bacillus_spp_as_source_of _Natural_Antimicrobial_Compounds_to_control_aquaculture_bacterial_fish_pathogens/links/5bec1f24928 $\overline{5} 1 \mathrm{c} 6 \mathrm{~b} 27 \overline{\mathrm{b}} \mathrm{de} 384 /$ Bacillus-spp-as-source-of-Natural-Antimicrobial-Compounds-to-control-aquaculture-bacte rial-fish-pathogens.pdf.

Schneider, O., Sereti, V., Eding, E.H \& Verreth, J.A.J. (2005). Analysis of nutrients flows in integrated aquaculture systems. Aquaculture Engineering. 32: 379-401.

Shea, D., Bateman, A., Li, S., Tabata, A., Schulze, A., Mordecai, G., Ogston, L., Volpe, J.P., Neil, F.L., Connors, B., Miller, K.M., Short, S. \& Krkošek, M. (2020). Environmental DNA from multiple pathogens is elevated near active Atlantic salmon farms. Proceedings . R. Soc. B. 287.

Snieszko, S.E. (1973). Diseases of fishes and their control in the U.S. The Two Lakes Fifth Fishery Management Training Course Report London (ed. J.K.S. Jansen

Stentiford, G.D. Neil, D.M., Peeler, E.J. Shields, J.D. Small, H.J. Flegel, T.W. Vlak, J.M. Jones, B. Morado, F. Moss, S. Lotz, J. Bartholomay, L. Behringer, D.C. Hauton, C. Lightner, D.V. (2012). Disease will limit future food supply from the global crustacean fishery and aquaculture sectors. Journal of Invertebrate Pathology. 110: 141-157.

Sumi, C.D., Yang, B.W., Yeo, I.-C., \& Hahm, Y.T. (2014). Antimicrobial peptides of the genus Bacillus: a new era for antibiotics. Canadian Journal of Microbiology. 61: 93-103.

Tyagi, V.K., Chopra, A.K. Kazmi, A.A. \& Kumar, A. (2006). Alternative Microbial Indicators of Faecal Pollution: Current Perspective. Journal of Environmental Health Science and Engineering. 3: 205-216.

Vital, M., Stucki, D., Egli, T. \& Hammes, F. (2010). Evaluating the Growth Potential of Pathogenic Bacteria in Water. Applied and Environmental Microbiology. 76: 6477-6484.

Vouga, M. \& Greub, G. (2016). Emerging bacterial pathogens: the past and beyond. Clinical Microbiology and Infection. 22:12-21 
Walters, G.R. \& Plumb, J.A. (1980). Environmental stress and bacterial infection in channel catfish, Ictalurus punctatus Rafinesque. Journal of Fish Biology. 17: 177-185

Wu, S., Wang, G., Angert, E.R., Wang, W., Li, W. \& Zou, H. (2012). Composition, Diversity, and Origin of the Bacterial Community in Grass Carp Intestine. PLoS ONE 7(2): e30440.

Yanbo, W. \& Zirong, X. (2006). Effect of probiotics for common carp (Cyprinus carpio) based on growth performance and digestive enzyme activities. Animal Feed Science and Technology. 127:283-292. 